\title{
A COGNITIVE APPROACH TO DEFINITENESS: FROM SPECIFIC TO GENERIC
}

\author{
KEISUKE KoGA \\ Yamaguchi University
}

\begin{abstract}
This paper presents a study of the meaning of the English definite article using the framework of Cognitive Grammar developed by Ronald Langacker. What we focus on in particular is two kinds of non-specific usage: the generic definite and what we call the role definite. Our analysis in terms of Cognitive Grammar constructs and the newly introduced Identity Model will show that those definites designate imaginary individuals set up for expressive purposes. Definiteness itself is defined as the speech-act participants' mental access to the identity of the designatum.*
\end{abstract}

\section{Introduction}

The present paper deals with the English definite article with special reference to its non-specific areas, using the framework of Cognitive Grammar (Langacker (1987a, 1991)). Section 1 shows how insufficient amount of attention has been paid to the non-specific uses of the definite article, especially what I call role definites. Section 2 briefly introduces the theoretical constructs of Cognitive Grammar that are needed to treat definiteness, with my own proposal for capturing what underlies the imagery of definiteness. Sections 3 and 4 show how the device can accommodate the role definite and the generic definite respectively.

* This paper has developed out of the presentation I made at the Eighth National Conference of the English Linguistic Society of Japan (November 17-18, 1990, Rikkyo University), which seems to have presented problems rather than a solution to them. I would like to thank a number of people I discussed this topic with; Prof. Yoshikiyo Kawase (Seinan Gakuin University), among others, who gave me useful comments and warm encouragement.

English Linguistics 9 (1992) 152-175 -152-

(C) 1992 by the English Linguistic Society of Japan 


\section{Non-Specific Uses of the Definite Article}

The meaning of the English definite article has received a considerable amount of attention in grammatical studies of English. Most of the attention, however, has been paid to the question of how to define the specific use of the definite article such as (1)-(3)

(1) Fred was discussing an interesting book in his class.

a. I went to discuss the book with him afterwards.

b. He is friendly with the author.

( 2 ) What's wrong with Bill?

Oh, the woman he went out with last night was nasty to him.

( 3 ) Pass me the bucket, please.

((1)-(3), Hawkins (1978))

What is common to them and similar examples, anaphoric or otherwise, is that the referent is a particular object identified in some way by both the speaker and hearer (Hawkins (1978, 1991), Chesterman (1991)).

By contrast, surprisingly insufficient amount of consideration has been given to non-specific areas of definiteness. Among them, the generic definite is an area that has received relatively more attention. Recently, for instance, Heyer $(1985,1990)$ proposed the distinction between kind generics and default generics.

Kind generics

(4) The lion is a species

( 5 ) The dinosaur has been extinct since the Mesozoic.

(6) The muskrat was imported into Europe in 1906.

Default generics

(7) The lion is a beast of prey.

( 8 ) The Scotsman drinks Whisky.

What underlies the distinction is the fact that the former type takes either kind-level predicates or stage-level predicates and the latter takes individual-level predicates. (See Carlson (1977) for this three-fold division of predicates.) Heyer claims that both types of definite generics refer to what he calls generic individuals, which have two kinds of properties, namely those that apply to the kind itself $((4)-(6))$ and those that determine certain properties of all or typical members of the kind ((7)-(8)).

Although there seems no doubt that the definite generic refers to something like a kind-as-individual, Heyer is not explicit as to in what sense we can speak of the kind as an individual. The generic individual, if there is such a thing, seems to be inherently unbounded in space and time 
in the sense that it can cover a potentially infinite expanse of spaciotemporal area that a single instance can never reach by itself, as illustrated in (9)-(10).

(9) The horse lives in many parts of the world.

(10) The horse has been domesticated for thousands of years.

Needless to say, a single instance of horse can never live in many parts of the world or have been domesticated for thousands of years. Normally, we can speak of individuals when it is inherently bounded in space (desk, house, town, country, etc.), in time (minute, hour, day, week, etc.) or in both (event, accident, meeting, etc.), or other domains. Then, as long as we insist that the generic individual is an individual in some sense, we have to specify where the individuality comes from.

Related to the problem of individuality is the fact that a mass-noun generic cannot refer to the whole kind when it is in the definite form.

(11) a. Gold is traded in many parts of the world.

b. The gold is traded in many parts of the world.

The gold in (11b) cannot refer to gold in general but only to a specified amount or type of gold. Heyer would argue that this is because the kind $<$ gold $>$ is not an individual. While we know that speakers of English do not think of a specific portion of gold as an individual, we can properly ask why they do not see the kind as such either. No linguists who have dealt with the definite generic, as far as I know, have provided any clear account for the puzzle.

Another thing to be noted is that most grammarians who have studied the meaning of the definite article have seen it in dichotomy: it may be specific, or else generic, period. ${ }^{1}$ There is, however, an area of definiteness which does not seem to belong to either category.

(12) In many of the cases observed, the wife was easily depressed when the husband held an important office.

(13) In most of the cases examined, the dog was expensive when it was of a rare breed.

(14) In all 54 cases examined the wolf was intelligent when it had blue eyes and stupid when it had brown eyes.

(15) (The social workers sometimes told the pregnant women about

\footnotetext{
${ }^{1}$ For instance, Hawkins (1978), Platteau (1980), Heyer (1985, 1990), Thrane (1985), Chesterman (1991), and Ojeda (1991), to take recent studies alone.
} 
the dangers of smoking.) When the mother understood this, she sometimes gave up smoking, but in most cases she did not.

((12)-(15), Declerck (1988), my emphasis)

The wife, the husband, the dog, the wolf, and the mother in (12)-(15) cannot be called specific definites, because they all involve, and in that sense "refer to," multiple instances of respective classes of objects despite the singularity in form. Nor can they be called generics, considering that they are only concerned with limited numbers of instances. Whereas they are used in talking about something significant about the cases concerned, they suggest nothing significant about the kinds <wife>, <husband>, $<\mathrm{dog}>,<w o l f>$, and $<$ mother $>$. Since there is no established term that refers to this type of definite NP, I will call it the role definite, borrowing the term role and its basic notion from Fauconnier (1985). ${ }^{2}$

One might ask whether this type of NP can only occur in special constructions such as atemporal when-clauses in (12)-(15) (Carlson (1979), Declerck (1988)). Actually, it can appear more freely.

(16) I hate his detective stories because the criminal always commits suicide at the end.

(17) The absence of positive role models may also cripple black boys' development. Nationally, $55.3 \%$ of black families with children under 18 are maintained by the mother, many of them living in inner cities. (TIME (May 21, 1990:51), my emphasis)

(18) These days, marrying an American serviceman in Korea can require as much courage as love. Korean women strolling hand in hand with Americans are often subjected to muttered insults from other Koreans and most mixed marriages are opposed by the wife's family. (TIME (July 30, 1990:51), my emphasis) Role definites, it seems, can occur fairly freely where something general is stated about multiple cases or situations of the same sort, regardless of

2 Apart from Hintikka and Kulas (ex. (24) below), as far as I know, only Fauconnier (1985:41) mentioned the phenomenon, only briefly, citing the following.

(i ) Every man built himself a house. In John's case, the house was a two-story brick structure; in Harry's case, the house was a huge glass frame design; in most cases, the house was conceived for four or five.

Interestingly, Declerck (1988), who supplied (12)-(15), did not say a word on the definite NP. Neither Carlson (1979:93), whose example (ii) first called my attention to the phenomenon.

(ii) Restaurants are bad places to eat when the head waiter is a greedy person. 
the construction they appear in.

It might be objected that (16)-(18) are examples of the associative anaphoric such as (1b) (book-author). It is true that one can feel something like anaphoricity with the combinations like detective story/ criminal, family/mother, and marriage/wife. But why do they come up in the singular form while multiple instances are involved, as the plurality of the "trigger" words indicate? This is suggesting that the problem includes something more than simple anaphoric reference.

Although it seems clear that the role definite would come somewhere between the specific and the generic definite if we drew an overall picture of definiteness, no one has shown how to characterize the former in relation to the latter two. And that is exactly what we try to do in the sections below.

\section{The Framework of Cognitive Grammar and Definiteness}

\subsection{The Conception of Nouns}

In Cognitive Grammar, the meaning of a linguistic expression, which is thought to be conceptualization, is characterized in reference to one or more cognitive domains. Potentially, any kind of human cognition can be a cognitive domain and there is no absolute limitation. While most domains directly relevant to semantic characterization are of complex sorts, some domains are basic in the sense that they cannot be reduced to more primitive ones (e.g. time, space, color sensation, pitch sensation, emotions, etc.).

The set of domains needed to characterize the meaning of an expression is called its base. The substructure of the base that is directly designated by the expression is called its profile. The meaning of arc, for instance, necessarily makes reference to the concept [CIRCLE], of which it is a part; in that sense, [CIRCLE] provides the base for the concept [ARC] and a segment of that base is the profile (designated substructure) of the expression arc. [CIRCLE], in turn, is characterized as a type of configuration in two-dimensional space. The base of circle, therefore, is the twodimensional space and the profile is a round configuration in it.

Nouns designate a region in some domain(s). A region means a set of conceptually interconnected entities. For example, the names of physical objects (desk, chair, bicycle, etc.) designate configurations in physical space. Moment, instant, and period designate regions in time. Second, 
minute, hour, day, etc. designate conventionalized units in time. Color terms designate particular regions in the domain of color sensation or "color space."

The count/mass distinction of nouns is made in reference to the notion of bounding. A count noun designates a region inherently bounded in its domain of instantiation. The domain of instantiation is a cognitive domain where the profile of the noun has observable instances (e.g. physical space for physical objects and material substances, time for temporal entities, etc.). The nouns cited above are count nouns because they are conceptualized as having inherent boundaries. The bounding, however, need not be sharply demarcated or completely closed. The profile of month is more precisely bounded than that of season. Center (of the room) has only fuzzy boundaries. The boundaries of river and road are usually partly unclosed so that one often can not tell where they begin or end.

A mass noun, by contrast, designates a region that is not inherently bounded in its domain of instantiation. Liquids like water, coffee, milk, etc. and substances like iron, gold, silver, etc. are typical examples. Though they exist in the real world in the form of bounded portions, they are conceptualized as having no built-in boundaries. It is ultimately a matter of construal what kinds of things are categorized as a mass, though it is often motivated by objective reality.

\subsection{Type, Instance, Identity, and Definiteness}

Since language refers to things and situations through their type conceptions, we cannot go without touching upon how to incorporate the type/instance distinction into Cognitive Grammar. Since Cognitive Grammar assumes that everything language evokes is conceptual, both type and instance are assumed to be conceptions we have in mind when we speak about something. Moreover, they are in some ways analogous; both conceptions, for instance, invoke the domain of instantiation to specify what they profile. As Langacker (1991:57) notes, both the type conception and the instance conception of cat "evoke and profile the conception of a furry creature that occupies physical space and has a certain configuration in this domain (i.e. a shape)." Then, how can we characterize the difference between them? Langacker's suggestion in this respect is that "an instance (but not a type) is thought of as having a particular location in the domain of instantiation." The type conception of cat uses its domain of instantiation to specify what shape it has in physical space but does not associate 
it to a certain location. The instance conception, on the other hand, places a specific cat in some particular location in the domain. Though the instance can move around, it occupies only one place at a given moment.

Cognitive Grammar also assumes that the type/instance distinction bears directly on the grammatical distinction between noun and nominal. A noun, however modified and complex, designates a type (or subtype). A nominal (or NP), by contrast, designates an instance whose epistemic relationship to the speech situation (or, more accurately, the speaker's conception of the speech-act participants and the immediate environment) is specified. ${ }^{3}$

(19) a. white cat which has a long tail

b. a/the white cat which has a long tail

(19a), with nothing that indicates whether the cat is an entity known to the speech-act participants, is an ungrounded complex noun designating a complex type. (19b), with a grounding expression, which specifies the epistemic status of the cat with respect to the speech situation, is a nominal and designates an instance of that type. ${ }^{4}$

Now, while we have seen the theoretical device in Langacker's framework needed to deal with definiteness, let us diverge a little from him and introduce an Idealized Cognitive Model that I think is needed to accommodate non-specific usages of the definite article. Idealized cognitive models are man's idealized views of how things and situations in the world are structured (Lakoff (1987)). They are simplified and highly prototypical hypotheses about the world that motivate and at times constrain the meanings of words and grammatical structures. ${ }^{5}$ They are, accordingly, sometimes in conflict with how the objective situation really is. I assume further that they can be modified in various ways when it is motivated by expressive purposes.

${ }^{3}$ The conception of the speech situation is called Ground in Cognitive Grammar. See Langacker $(1985,1991)$ for the detailed discussion of what it is like and how it works in the overall framework.

${ }^{4}$ The so-called determiners (articles, demonstratives, possesives, and quantifiers) roughly correspond to grounding expressions for nominals. See Langacker (1991: Ch. 2-Ch.4).

5 Idealized cognitive models (the billiard ball model, the stage model, the epistemic models, etc.) play crucial roles in Cognitive Grammar, especially when it characterizes such fundamental notions as transitivity, grammatical relations, case, voice, tense, and modality. See Langacker (1991) for detailed discussions about them. 
The model to be introduced here is the Identity Model, defined as follows:

(20) Identity Model

Every bounded instance, whether it is an individual or a mass, has its own identity, which distinguishes it from all the other instances of the same type. The type itself is devoid of identity.

I claim that (20) underlies all conceptualizing activities of English speakers and fundamentally determines how the article system works.

Applied to nominal semantics, (20) states that every instance individual (designated by a singular count nominal) is regarded as having an inherent identity. It may be helpful to imagine that each individual (person, dog, chair, car, etc.) has a "uniform number" distinct from the other instances of the same type, as a baseball player does, and one can single out a particular individual by reference to its "number." A mass, on the other hand, has no identity unless it receives some kind of external bounding in the domain of instantiation. Water, for example, cannot be regarded as having an identity as long as it is conceived of as something that has potentially any size of expanse. Once its expanse is limited by some boundaries, we can think of its identity and refer to it as the water in the cup/in the lakelthat I drank just now, etc. A replicate mass, designated by a plural noun phrase, behaves in the same way as a mass. Although it is derived through the cognitive process of replication from the type conception of an individual (Langacker (1991:74-81)), we assume that indefinitely replicated individuals (designated by bare plural forms, dogs, chairs, cars, etc; cf. Carlson (1977)) cannot have their identity unless externally bounded; once they are somehow bounded (some dogs, the dogs, these dogs, etc), they will have a collective identity as well as the individual identities of the members.

The notion of identity thus defined, the meaning of the definite article can be stated in the following terms:

(21) Definite Article

The definite article indicates that both the speaker and hearer have mental access to the identity of an instance conception as well as its type specification, whether it is an individual or a mass. ${ }^{6}$

${ }^{6}$ In contrast, the meaning of the indefinite article can be defined as indicating that 
To have mental access to the identity of something means to be able to distinguish it from all the other instances of the same type. In this sense, the anaphoric use is the prototypical usage of the definite article because it profiles an instance conception introduced in the previous discourse, adding to its base the conception of the mental access to its identity. ${ }^{\text {? }}$

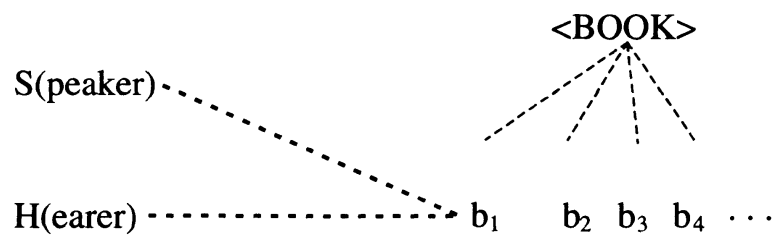

Generally, appearance in the previous discourse makes it possible for the speech-act participants (the hearer, in particular) to distinguish the instance conception of the book from all the other instances of the type <book>, thereby enabling them to have mental access to its identity. Consequently, the speaker can refer to the book as the book. The other uses of the specific definite (the associative use, the immediate-situation use, etc.) vary only in what kind of condition serves to make the mental access possible.

It should be noted that the possibility of the mental access alone does not always make the use of the definite article actual.

(22) J: "I left the sum of fifty thousand pounds to be held in trust for Ruby Keene until she was twenty-five, when she would come into the principle."

H: "That's a large sum of money, Mr. Jefferson."

$\mathrm{J}$ : "In these days, yes, it is."

$\mathrm{H}$ : "And you were leaving it to a girl you had only known for a few weeks?"

(A. Christie, The Body in the Library, my emphasis) The first and third speaker, namely Mr. Jefferson, and the second and fourth speaker, Superintendent Harper, are both fully aware that $a$ girl you had only ... refers to Ruby Keene, a girl who was murdered. To express

both seech-act participants have mental access only to the type specification of the instance conception of an individual. This means, in other words, that the speaker and hearer are conceptualized as viewing the instance only in terms of the type specification it has (cf. ex. (25) below).

${ }^{7}$ Grounding expressions do not pofile the epistemic relationship itself; instead, they profile the instance conception, adding the epistemic relationship to the base of the nominal. See Langacker (1985). 
his surprise at Jefferson's idea of leaving a large sum of money to a girl he has known for such a short time, Harper refers to her in terms of what kind of type specification she has, because what matters here is not the identification of the girl as Ruby Keene but the fact that she instantiates the type < girl you had only ...>. Replacing the nominal with her or the girl you had ... would make it clearer: one could not feel the nuance of surprise "to such a girl?" any more. In general, it is up to the speaker in a particular situation to decide whether to refer to the mentally accessible entity as such. It is not a matter of rule but of subjective judgment and choice.

Details aside, uniqueness, familiarity, identification, inclusiveness, and other terms that have appeared in attempts to define the meaning of the definite article (cf. Hawkins (1978) and Chesterman (1991)) all try to capture roughly the same thing as the notion of the mental access to identity, each with its own emphasis on particular aspects of the process. In that sense, we can regard them as locutionary variants so far as we are concerned only with specific definites; after all, they all presuppose the normal state of the Identity Model. A step out of the area, however, will reveal that one cannot deal with definiteness without substantive hypotheses as to how the things referred to are structured and can be restructured in our mind.

\section{Role Definites}

With role definites such as (13), we have a paradox.

(13) In most of the cases examined, the dog was expensive when it was of a rare breed.

Whereas the definite singular form, on the one hand, suggests that it refers to one object, the "trigger" phrase most of the cases examined points to the involvement of multiple dogs. It is not, however, that the situation is totally unreasonable. (13) implies that the relevant cases are conceptualized in parallel fashion so that each case includes only one dog. The same is true of (12) and (14)-(15). Let us call this mode of conception a parallel-case structure or image.

To deal with this apparently paradoxical referential structure, we assume that the following modification holds in the Identity Model.

(23) Role Establishment

Given a parallel-case structure where individuals of the same kind play the same role in separate cases, we can abstract that 
role away from the individuals and establish it as a special instance individual with a role identity.

According to the normal state of the Identity Model, specific individuals alone have an identity. Role Establishiment makes temporary modification to it and produces a single individual (a role) equipped with an identity that consists of the type specifications of the relevant individuals. In other words, the role individual is an imaginary individual set up pariticularly for describing what kind of role the relevant type is playing through the activity of an instance individual in each case of a parallel-case structure. ${ }^{8}$

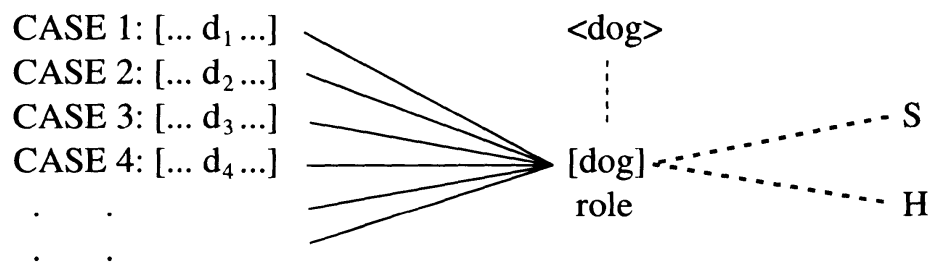

The role individual is an individual because its identity has inherited individuality from the type specification of the instance individuals concerned. The role definite nominal profiles the role individual, relegating the rank-and-file instances into the base of the nominal. That the profiled role is unique in the current discourse (since no other such role is established) ensures that the speaker and hearer have mental access to its identity, allowing the definite singular form. The same applies to (16)-(18).

Since the Role Establishment is a modification of the normal state of the Identity Model, the speaker have to provide the hearer with something

${ }^{8}$ One anonymous reviewer suggested that the Mental Space framework by Fauconnier might serve to give a little more formalization to the concepts used here such as mental access and imaginary individual. There is no space for extended discussion, but of the concepts developed in Fauconnier (1985), only the role/value distinction (Ch. 2) was really helpful to me in that it gave me a notion that can capture the examples that fall in between specific and generic definites. As for the notion of mental access, all I can say for the moment is that it is intended to capture the mode of linguistic reference a person chooses when he/she refers to entities: given an instance individual, we can choose either the access to its type specification or the access to its instance specification (i.e. identity). I have little idea how the Mental Space theory would describe the notion. 
indicative or suggestive of the modification before he uses the role definite nominal. Thus, the "trigger" phrases must precede the use of the role definite nominal.

$\left(16^{\prime}\right) *$ The criminal always commits suicide at the end, so I hate his detective stories.

$\left(17^{\prime}\right) *$... Nationally, the mother maintains $55.3 \%$ of black families with children under $18, \ldots$

$\left(18^{\prime}\right) * \ldots$ and the wife's family opposes most mixed marriages. The awkwardness of $\left(16^{\prime}\right)-\left(18^{\prime}\right)$ is clearly due to the reversed order.

Another thing that follows from this characterization is that the role can survive as long as the parallel-case image is sustained in the discourse. This means that the "trigger" phrase can be outside the sentence in which the role definite nominal appears, like (24).

(24) Every marriage has its own problems. Sometimes the husband is the source of the problems, sometimes the wife.

(Hintikka and Kulas (1985: 63))

It is clear that the adverb of quantification sometimes helps preserve the parallel-case image introduced by the phrase every marriage. It should be noted in passing that examples like (24) suggest that one cannot treat the role definite in the same way as a simple bound-variable pronoun like its in Every marriage has its own problems.

One might ask why we should have such a peculiar individual in our conception when we really mean multiple individuals. The motivation for having Role Establishment, I think, lies in the fact that bounded plurality gives rise to ambiguity when it is predicated of something. It is well known, for example, that (25) is ambiguous between the distributive and the collective reading:

(25) The dogs are expensive.

It means either that each dog is expensive or that the dogs as a whole are expensive though each of them may not. But the ambiguity does not arise with the role definite nominal since it is based on a parallel-case image.

It is not that just any kind of parallel-case structure allows one to set up a role individual. Role Establishment also requires a significant generalization about the role that the individuals play in the cases concerned. This is directly demonstrated by the use of generality markers such as atemporal when-clauses in (12)-(15) and the adverb of quantification always in (16). It is also suggested by the fact that the number of the cases concerned, normally unspecified, can only be made explicit if it is numerous enough 
to allow one to talk about some general tendency over the cases.

(14) In all 54 cases examined the wolf was intelligent when it had blue eyes and stupid when it had brown eyes.

(17) ... Nationally, $55.3 \%$ of black families with children under 18 are maintained by the mother, many of them ...

(14) is a general statement about the wolves concerned suggesting the close (perhaps causal) relationship between the color of the eyes and the intelligence. (17) is not just a statement of fact but a generalization about the U.S. black families which indicates the general lack of male role models, namely fathers, for young black boys. If the number of the cases is too few to make any significant generalization, however, the role definite nominal is awkward.

$\left(14^{\prime}\right) *$ In these two cases, the wolf was intelligent when it had blue eyes and stupid when it had brown eyes.

$\left(17^{\prime}\right) *$ Only five black families with children under 18 are maintained by the mother.

I attribute this generality condition to the nature of the role identity. Because the identity of a role has inherited the type specifications of the individuals distributed in a set of parallel cases, it must include specification about what kind of general tendency the type has within this specific range of cases.

The last question in this section is concerned with the distinction between the role definite and the generic definite. Observe (26).

(26) The prescribed role of the man as "breadwinner" and the woman as housewife is changing. These changes include working mothers, "househusbands," and an increasing number of day-care centers for children. Yet traditional roles may be preserved even in households where the wife is working. (D. R. Levine and M.B. Adelman, Beyond Language, my emphasis)

Although it is obvious that the italicized definite nominal, based on a parallel-case image, is a role definite, it is not easy to argue that it is any different from the generic definite, because the discourse containing it has a generic flavor. (27)-(28) further demonstrate the difficulty.

(27) Vampires dreaded only daylight, which made them crumble to dust, a crucifix, and garlic. They could be killed by driving a stake through their hearts or by shooting them with a silver bullet. Stabbing them with a silver knife would probably have the same effect, if the vampire, with its supernatural strength, 
didn't wrest the weapon from your fear-paralyzed hand and use it on you.

(Peter Limburg, Stories Behind Words, my emphasis)

(28) There are three steps to learning any language. The first step is to recognize that the sounds being made actually apply to some physical object or condition. If babies say "BabaMamaGaga," mothers in one country will rejoice that they said "Baba," in another, because they said "Mama," and in a third, because they said "Gaga." The baby itself is not conscious that the sounds it is making apply to a particular person. Only through the constant reinforcement of the mother does the baby imagine that there is some connection between the sound "mama" and a particular human being.

(Carl Becker, Communication: East and West, my emphasis) Both of the passages describe a generalized situation. What matters in (27) is this: the description started out with generic sentences including plural forms vampires, they, and them, but as it turns to a fighting scene, the plurality goes out and singularity comes in. Why? Because it would be clumsy to describe the fight by presenting multiple cases and objects directly, as illustrated in (27').

$\left(27^{\prime}\right)$..., if vampires, with their supernatural strength, didn't wrest weapons from your fear-paralyzed hands and use them on you.

$\left(27^{\prime}\right)$, if not unacceptable, may mean something different from (27): multiple vampires vs. multiple persons in one scene, that is, a collective reading. The definite singular form in (27) is employed to put the one-toone fighting relation into relief, while the whole sentence is a general statement which constitute a part of a generic discourse. One can think of the baby and the mother in (28) analogously.

The problem is whether they are really different from the so-called definite generic, or the same. That is what we will discuss in the next section.

\section{Generic Definites}

In Section 1, we agreed with Heyer that the generic definite designates a generic individual. It is our turn to provide cognitive characterization of what that individual is like. 
(29) Generic Individual Establishment

Given a macroscopic setting where potentially infinite number of instances of the same type are playing, distributively or collectively, a role which is significant for the characterization of the type, we can imagine that a single large-scale individual with a type identity is playing the role.

A type identity is an identity that distingishes the type itself from any other instance subsumed under it. Although the type itself is assumed to be devoid of identity in the normal state of the Identity Model, it is conceptualized as a special instance distinguished from rank-and-file instances when it is participating in a particular relationship significant for type characterization. The underlying intuition is that the generic individual is not a type conception that is simply "out there" waiting to be referred to, as many linguists explicitly or implicitly assumed; the type itself cannot be put in nominal profile unless it is imaginarily set up as an abstract individual. ${ }^{9}$ Because this individual is an instance, it must have a particular location in its domain of instantiation (see Section 2.2). In this case, the domain of instantiation is a macroscopic setting and the "particular location" means the role this individual plays in a relationship significant for the type's characterization. The definiteness is due to its uniqueness in the setting: what is put in profile is the generic individual that subsumes all the relevant instances in its base.

One might ask why we should have such an arrangement in order to refer to a type. The purpose of setting up a generic individual, I think, is to refer to macroscopic situation without resorting to the image of plurality. Since macroscopic situation can include an infinitely large number of instance individuals of a type, as we noted in (29), without a generic individual one would have to use the image of unbounded plurality, normally expressed by a bare plural nominal, to refer to it. Unbounded plurality, however, sometimes cannot convey genericness unambiguously. Consider the following pair.

(30) a. Hurricanes arise in this part of the Pacific.

(Carlson (1989: 170))

${ }^{9}$ Recall that Cognitive Grammar assumes that a type conception is designated by a noun, not by a nominal, which desingnates an instance. An ungrounded type conception only occurs as a component of a nominal, like cat in a cat lover and dog in this kind of dog. 
b. The hurricane arises in this part of the Pacific.

(30a) is ambiguous between two readings: one is that there arise hurricanes around here (this is a significant property of this part of the Pacific) ${ }^{10}$ and the other is that it is a significant property of hurricanes that they arise around here. (30b) only gives rise to the second reading. This is because a bare plural designates a replicate mass that can expand indefinitely without regard to its significance for type characterization, but a generic individual can exist covering a large-scale area only insofar as the area guarantees a description significant for type characterization. In other words, while the generic individual is specifically intended for generic description, the replicate mass designated by a bare plural is not.

Another sort of ambiguity that can arise with bare plurals is the collective/distributive ambigity we saw in (27'), where the one-to-one interaction manifest in (27) is blurred by the use of bare plurals. This point, however, can be made only if we assume that (27)-(28) are examples of generic definite. Then, we now have to answer the question posed at the end of the last section: what is the difference between the generic definite and the role definite?

The difference lies in the necessity of parallel-case image: the role definite requires the image but the generic definite does not, as long as it is placed in a macroscopic context, though it does not exclude the image either. It comes as no surprise, then, that it is quite difficult to draw a clearcut borderline between the two. As long as the latter retains an underlying parallel-case image, the distinction between them is nothing more than a matter of degree: the latter is simply an extention of the former along the cognitive scale of macroscopicness, which in turn is measured for its significance for type characterization. Observe another example.

(31) Being a housewife is nothing to be ashamed of in Japan. Because most husbands leave their salaries and children entirely in the hands of their wives, women have wide-ranging responsiblities. It was not always thus. Traditionally, wives

${ }^{10}$ This reading is due to the fairly limited expanse of the location (this part of the Pacific) and the temporal open-endedness of the description. The former prevents the sentence from being significant to the characterization of the type <hurricane>. The latter induces an intensional interpretation in which potentially infinite number of hurricanes are scattered along the time axis. See Carlson (1989) for a detailed study of this and similar examples. 
and children blindly obeyed the father as ruler of the roost.

(TIME (December 3, 1990: 41), my emphasis)

The italicized definite nominal is a role definite, on the one hand, to the extent that it is based on a parallel-case structure where the image of one father being in one case holds. The nominal is also a generic definite, on the other hand, in the sense that the individual it designates is set up in a macroscopic setting (i.e. traditional Japan) specified by context and could be considered significant in contribution to the characterization of the type $<$ father $>$.

In this light, Heyer's distinction between kind generics and default generics captures only a part of the whole picture. Heyer's examples are all sentences with the generic nominal in the subject position; he has nothing to say about those examples with the generic definite in oblique positions, because his distinction is based on what kind of predicate is attributed to the subject (i.e. kind/stage-level vs. individual-level). From our point of view, the distinction is a special case of a more general distinction based on the existence of parallel-case image. Furthermore, what licenses the generic definite, whether it is a kind or default one, is a macroscopic setting in which the generic individul participates in a relationship significant for the characterization of the type, the point he failed to mention.

One question naturally arises here concerning the notion of macroscopicness: how macroscopic is macroscopic enough to be interpreted as generic? Unfortunately, macroscopicness is a matter of degree and subjective evaluation; it can by no means be objectively specified. Consider (32), from Thrane (1980: 200).

(32) a. The castle is a prominent feature of Welsh scenery.

b. The castle is a prominent feature of Edinburgh' scenery. (32a), Thrane notes, "will probably always be given a generic interpretation," but (32b) "could only be non-generic." One cannot share the judgement without knowing what Wales and Edinburgh are like and how they are different from each other. Nor can we ever specify how many castles in how much size of land can serve as a setting for interpreting the castle in such sentences as generic.

All we can do is to provide specific forms of macroscopicness that can induce Generic Individual Establishment. First, as we have seen in (9)-(10) and (31)-(32), descriptions with a spatio-temporal expanse that we generally consider to be large enough for type characterization can serve as generic settings. It should be noted that temporal macroscopicness includes 
those settings in which specific events of historical importance are described.

(33) The horse came to America with Columbus.

(34) Explorers first saw the buffalo in 1517.

((33)-(34), Carlson (1977:278))

As Carlson notes with (33)-(34), they do not make sense unless there had been no horses in America before they came with Columbus and no buffalo had been seen before 1517 (at least by European explorers), if they are to be interpreted as examples of the generic definite. The event in profile is something that occurred at a particular place at a particular time, and the instances involved are a limited number of particular individuals. But the event and instances are evaluated with respect to their historical importance against a macroscopic time span, and to the extent that those instances are seen as having played a historic role for the species, they are conceptualized as a generic individual. A comparison with the following will confirm that.

(35) The horse arrived on my doorstep yesterday.

(Carlson (1977: 279))

The horse in (35) as a generic definite is "exceedingly strange" even if "there has never been a horse on my doorstep before."

Second, even without such a particular expansion of time and space, it seems enough to have a generalized description in which the imaginary individual functions in a significant manner for the type's characterization. (27)-(28) are cases in point. Let us take a look at a few more examples.

(36) When we travel abroad we are immediately impressed by the many ways buildings, homes, and cities are designed. The division and organization of space lend character and uniqueness to villages, towns, and cities. Yet architectural differences may also cause confusion or discomfort for the traveler.

(Beyond Language, my emphasis)

(37) In a normal country, currency is more than just a medium of exchange between a buyer and a seller: a dollar bill or a thousand-yen note is a contract between the individual and the state. The citizen does his part by producing and consuming. The government's end of the bargain is to ensure what economists call a stable standard of value - a sound currency - for the transactions of life.

(TIME (March 16, 1992: 12), my emphasis) 
(36) describes a possible psychological reaction of the imaginary traveler to architectural differences in a generalized journey setting. Note also that here the traveler is interchangeable with the bare plural travelers, which would evoke a slightly different image of the situation with its plurality. (37) is crucially different in that respect. It is an established part of our knowledge that a government has control over a huge number of citizens. Given that the setting is a generalized image of one country (a normal country), we can replace the citizen with citizens, but a replacement of the government with governments will yield awkwardness.

Another important factor emerging in (37) is a contrast between two or more types in a generalized setting. In fact, such a contrast contributes to the conception of each type as an individual. The following example is more salient in this respect.

(38) Even on a clear night, astronomers cannot see forever. Light from the stars is diffused and distorted by the earth's atmosphere. To the casual stargazer, that produces a beautiful twinkle, but to the astronomer it is a tragic blur.

(TIME (April 16, 1990: 44), my emphasis)

(38) is reminiscent of the "superset" theory maintained by Plateau (1980) and a number of other linguists, because the existence of a superset (or superordinate type) strongly suggests that one type is contrasted with other types that belong to the same superset.

(39) A: What do you know about the koala?

B: The koala lives in Australia. It eats eucalyptus leaves.

Plateau (p.118) says "in this case the koala means: not the antilope, not the panda bear, not the chinchilla, but the entity that could be called koala," implying the existence of the superset <animal>. It is certainly true that an explicit mention of a superset makes it easier to obtain the generic definite, even if it is a non-anminal example:

(40) Facial expressions carry meaning determined by contexts and relationships. For example, the smile, which is typically an expression of pleasure, has many functions.

(Beyond Language, my emphasis)

But given the wide variety of non-animal nouns used in the examples so far, it seems better to think of the existence of a superset merely as one of the factors that can provide a macroscopic setting. Animal examples are priviledged to an extent in that they promptly remind us of the taxonomic 
hierarchy they belong to and their positions and functions in it. ${ }^{11}$ In addition, the superset theory, by itself, cannot account for the absence of definite generic with mass nouns. (Recall the difference between gold and the gold that we saw in (11).)

The discussion thus far teaches us an important lesson: given that the generic definite requires a macroscopic setting where a generic individual can play a significant role, it would be dangerous to study it by looking at single-sentence examples alone. A typical case is Carlson (1977:274-280).

(41) ? The tree gives off carbon dioxide at night.

(42) ? The primate is larger than the crustacean.

(43) ? The ruminant has cloven hoofs.

(44) ? The angel plays the harp.

(45) ? The house has several rooms.

(46) ? The airport is a busy place.

Citing (41)-(46), he suggests that certain nouns may be inherently prohibited from appearing in the generic definite form. Although he admits he found the tree and the primate can be generically used,

(47) No one really understands how the tree knows when to shed its leaves in the autumn.

(48) The primate is our main object of study in this class. ${ }^{12}$ he said he could not find any suitable contexts for the ruminant and the angel to be used generically. However, there are such examples.

(49) The primate comes far later than the crustacean in the history of evolution.

(50) The compound four-chambered stomach of the ruminant and the the process of rumination have excited the interest of agriculturalists and biologists generally from time immemorial.

(51) The angel as a winged creature is not original with Christianity. ((49)-(51), Ojeda (1991: 391-392))

(52) The house is the center of non-nomadic civilization.

(Thrane (1980: 203), slightly modified)

11 Incidentally, Kumayama (1985) provides examples of the generic definite with respect to human types, bodily parts and organs, family members, animals, plants, professions, musical instruments, etc.

${ }^{12}$ Generic definites can be used in the positions that refer to the object of study, discussion, talk, etc. because mental activity and locution can create any kind of setting one can imagine. 
(53) Among the tansportation sites that appeared in history, the airport is the largest and most complicated and, therefore, the most difficult to manage.

What differentiates (49)-(53) from (41)-(46), if any, is the implied existence of macroscopic settings, no matter how the settings are conceptualized. ${ }^{13}$ This means that (41)-(46) are judged to be awkward to the extent that they are interpreted as simply describing properties which have little to do with macroscopic settings, however general the properties are. (An extremely imaginative linguist might come up with some contexts that can make them acceptable, though.)

The final question to be answered in this section is why we have no definite mass-noun generic comparable to the count-noun one. The answer is fairly straightforward from our point of view, considering how things are structured in the Identity Model. Only individuals and bounded masses are allowed to have identity and only things with identity can have definite designation. This means a mass entity designated by a definite nominal is necessarily something externally bounded. To be generic in the ordinary sense, however, a mass entity must be unbounded. Consequently we have no mass-noun generics in the definite form.

One might ask why we have no special modification of the Identity Model that sets up the type conception of a mass as an abstract individual. The reason, I think, is that the motivation for such an operation is weaker with mass entities than with individuals. Inherently unbounded, a mass can be conceptualized as covering a large-scale area by itself, which is not the case with a normal individual. In addition, the ambiguity that can arise with plurality when one-to-one interaction is intended is irrelevant with mass entities, which are not subject to replication or one-to-one correspondence unless they are given some external bounding.

This means that when it is given some bounding, it is possible for a mass

${ }^{13}$ The same applies to Vendler (1967: 58-59), who cites (i).

(i) a. Euclid described the parabola.

b. ? Euclid described the curve.

He notes that the curve is too general to serve as a generic. It is true that in (ib) it is difficult to think of the class subsuming many different kinds of curve as a single individual. But the curve as a generic can be acceptable, as Ojeda (1991:391) illustrated:

(ii) The curve is not the shortest distance between two points.

Obviously, (ii) implies the contrast with the straight line. 
entity to be generically designated in the definite form, which seems to be borne out by the (a) sentences in the following.

(54) a. The blood picks up oxygen in the lungs and carries it to every part of the body.

b. Once out of the body, blood is very difficult to preserve without special chemicals and equipment.

(55) a. The snow keeps the seed warm in the ground.

b. Snow is the vapor of the atmosphere frozen by a current of cold air.

((55), Kramsky(1972: 53))

The difference between the (a) and the (b) examples is that the former, but not the latter, imply a generalized image of a bounded mass. Although strict treatment of these definite mass nominals requires further study, they are part of what is expected from our conception of the Identity Model.

\section{Conclusion and Further Issues}

Let me recapitulate the proposals made in this paper. Definiteness itself is defined in terms of the newly introduced Identity Model and the notion of the mental access to identity. The meanings of the role definite and the generic definite are explained in terms of imaginary restructurings of the Model to set up an abstract individual; one is based on a parallel-case image (the role definite) and the other is motivated for referring to macroscopic situation without resorting to plurality (the generic definite). Macroscopicness can only be measured subjectively for its significance to type characterization. In both cases of the non-specific definite, the definiteness is due to the imaginary individual's uniqueness in a special setting. This is because such an individual has nothing that can distinguish it from others except for the fact that it has been uniquely set up in an imaginary setting, as opposed to a normal individual, which has its own unique properties comprising its identity. With regard to mass-noun generics, the absence of the definite form follows from a peculiar nature of a mass: it has no identity by itself.

What has motivated me to adopt the framework of Cognitive Grammar is that it can deal properly with what lies behind our linguistic intuition about various manners of reference, in particular the conceptions of indivdiuality and boundedness. Without capturing them somehow in theoretical terms, the study of non-specific uses of definiteness would not go much further than the current level. The present study, I believe, will provide a serious 
first step toward re-examination of those areas in a new light, that is, in cognitive terms.

The present paper is of course not intended to provide an exhaustive picture of the meaning of the English definite article. The proposals concerning non-specific uses must be tested against nominals designating those entities that exist in non-concrete domains (e.g. properties and abstract things). The three forms of macroscopic setting (spatio-temporal macroscopicness, generalized settings, and taxonomic hierarchies) have to be further clarified in relation to genericness in general. The explanation for mass-noun generics is incomplete, considering that in French, German, and many other European languages, mass-noun generics must be definite (Kramsky (1972: 52-53)). These issues all call for independent studies in the future.

\section{REFERENCES}

Carlson, Greg N. (1977) Reference to Kinds in English, Doctoral dissertation, Univesity of Massachusetts. [Published by Garland, New York, 1980]

Carlson, Greg N. (1979) "Generics and Atemporal When," Linguistics and Philosophy 3, 49-98.

Carlson, Greg N. (1989) "On the Semantic Composition of English Generic Sentences," Properties, Types and Meaning II: Semantic Issues, ed. by Gennaro Chierchia, Barbara Partee, and Raymond Turner, 167-192, Kluwer Academic, Dordrecht.

Chesterman, Andrew (1991) On Definiteness: A Study with Special Reference to English and Finnish, Cambridge University Press, Cambridge.

Declerck, Renaat (1988) "Restrictive When-Clauses," Linguistics and Philosophy 11, 131-168.

Fauconnier, Gilles (1985) Mental Spaces, MIT Press, Cambridge, MA.

Hawkins, John (1978) Definiteness and Indefiniteness: A Study in Reference and Grammaticality Prediction, Croom Helm, London.

Hawkins, John (1991) "On (In)Definite Articles: Implicatures and (Un)Grammaticality Predictions," Journal of Linguistics 27, 405-442.

Heyer, Gerhard (1985) "Generic Descriptions, Default Reasoning, and Typicality," Theoretical Linguistics 12, 33-72.

Heyer, Gerhard (1990) "Semantics and Knowledge Representation in the Analysis of Generic Descriptions," Journal of Semantics 7, 91-110. 
Hintikka, Jaakko and Jack Kulas (1985) Anaphora and Definite Descriptions, Reidel, Dordrecht.

Kramsky, Jiri (1972) The Article and the Concept of Definiteness in Language, Mouton, The Hague.

Kumayama, Akihisa (1985) Eigo Kanshi Yoohoo Jiten (A Dictionary on the Usages of the English Articles), Taishukan, Tokyo.

Lakoff, George (1987) Women, Fire, and Dangerous Things, University of Chicago Press, Chicago.

Langacker, Ronald (1985) "Observations and Speculations on Subjectivity," Iconicity in Syntax, ed. by John Haiman, 109-150, John Benjamins, Amsterdam.

Langacker, Ronald (1987a) Foundations of Cognitive Grammar 1: Theoretical Prerequisites, Stanford Univeristy Press, Stanford.

Langacker, Ronald (1987b) “Nouns and Verbs," Language 63, 53-94.

Langacker, Ronald (1991) Foundations of Cognitive Grammar 2: Descriptive Applications, Stanford University Press, Stanford.

Ojeda, Almerindo (1991) "Definite Descriptions and Definite Generics," Linguistics and Philosophy 14, 367-397.

Platteau, Frank (1980) "Definite and Indefinite Generics," The Semantics of Determiners, ed. by Johan Van der Auwera, 112-123, Croom Helm, London.

Thrane, Torben (1980) Referential Semantic Analysis: Aspects of a Theory of Linguistic Reference, Cambridge University Press, Cambridge.

Vendler, Zeno (1967) Linguistics in Philosophy, Cornell University Press, Ithaca.

Faculty of Liberal Arts

Yamaguchi University

Yoshida, Yamaguchi-shi

Yamaguchi, 753 